\title{
FOURIER TYPE AND COMPLEX INTERPOLATION
}

\author{
MARIO MILMAN
}

\begin{abstract}
Using Fourier type arguments we provide a very simple proof of recent results on complex interpolation of $H^{p}$ spaces and martingale $H^{p}$ spaces. The same method gives a new result on complex interpolation of Sobolev spaces.
\end{abstract}

In a recent article (cf. [4]) S. Janson and P. Jones obtained a description of the complex method of interpolation for $H^{p}$ spaces and martingale $H^{p}$ spaces. Some of their main results are summarized in the following (for definiteness all our spaces (except the martingale spaces) are based on $\mathbf{R}^{n}$ ).

THEOREM A. (i) Let $X_{0}$ be either $H^{1}$ or $L^{1}$ and let $X_{1}$ be either $L^{\infty}$ or BMO; then $\left(X_{0}, X_{1}\right)_{\theta}=L^{p_{\theta}}, 0<\theta<1,1 / p_{\theta}=1-\theta$.

(ii) Let $X_{0}$ be either $M H^{1}$ or $M L^{1}$ and let $X_{1}$ be either $M L^{\infty}$ or $M B M O$; then $\left(X_{0}, X_{1}\right)_{\theta}=M L^{p_{\theta}}, 0<\theta<1,1 / p_{\theta}=1-\theta$.

The purpose of this paper is to provide a very simple proof of this theorem. Moreover, the method we use also yields the following interpolation theorem for Sobolev spaces.

THEOREM B.

$$
\left(W_{1}^{k}, W_{p}^{k}\right)_{\theta}=W_{p_{\theta}}^{k}, \quad \frac{1}{p_{\theta}}=\frac{1-\theta}{1}+\frac{\theta}{p}, \quad 1<p<\infty, \quad k \in \mathbf{N} .
$$

The novelty here is that we can include the extreme end point space $W_{1}^{k}$.

Our method consists of observing that by Wolff's theorem (cf. [10]) we reduce ourselves to check that we get the right results at each end point separately. In this situation we can avail ourselves of Fourier type arguments (cf. [8]) to reduce everything to real interpolation of the spaces involved, where the corresponding results are well known and easy to prove.

I say that a Banach space $A$ has type $p, 1 \leqslant p \leqslant 2$, if the vector valued Fourier transform maps $L^{p}(A)$ into $L^{p^{\prime}}(A), 1 / p+1 / p^{\prime}=1$. It is easy to check that every Banach space has type 1 and every Hilbert space has type 2 .

The importance of this notion is given by the following theorem of J. Peetre [8].

THEOREM C. Let $\left(A_{0}, A_{1}\right)$ be a Banach couple such that the type of $A_{i}$ is $p_{i}, i=0,1$. Then, $\left(A_{0}, A_{1}\right)_{\theta, p_{\theta}} \subseteq\left(A_{0}, A_{1}\right)_{\theta}$, where $0<\theta<1,1 / p_{\theta}=(1-\theta) / p_{0}+\theta / p_{1}$.

Received by the editors September 10, 1982 and, in revised form, January 12, 1983.

1980 Mathematics Subject Classification. Primary 46M35, 46E35, 42B30.

Key words and phrases. Fourier type, $H^{p}$ spaces, Sobolev spaces, interpolation spaces. 
Consider the proof of Theorem A(i). First we interpolate $\left(H^{1}, L^{2}\right)_{\theta}$. Since $H^{1} \subset L^{1}$ we trivially have $\left(H^{1}, L^{2}\right)_{\theta} \subset L^{p_{\theta}}, 1 / p_{\theta}=1-\theta / 2$. On the other hand, since the type of $H^{1}$ is 1 and the type of $L^{2}$ is 2 , we get, by Theorem $\mathrm{C}$, $\left(H^{1}, L^{2}\right)_{\theta, p_{\theta}} \subset\left(H^{1}, L^{2}\right)_{\theta}$. Thus $\left(H^{1}, L^{2}\right)_{\theta}=L^{p_{\theta}}, 1 / p_{\theta}=1-\theta / 2$ by [9]. Now, $\left(L^{q}, B M O\right)_{\theta}=\left(L^{q}, L^{\infty}\right)_{\theta}=L^{q_{\theta}}, 1 / q_{\theta}=(1-\theta) / q$, for $1<q<\infty$, by [3]. Therefore we obtain the first part of Theorem A by using Wolff's theorem to fuse these end point results.

For the martingale part we proceed in the same way. This time we use the known results on real interpolation of martingale $H^{p}$ spaces $(\mathrm{cf} .[5,6])$ together with the following.

LEMMA.

$$
\begin{aligned}
\left(M L^{p}, M B M O\right)_{\theta}=\left(M L^{p}, M L^{\infty}\right)_{\theta} & =M L^{p_{\theta}}, \\
& 0<\theta<1, \quad 1<p<\infty, \quad 1 / p_{\theta}=1-\theta .
\end{aligned}
$$

This result can be proved essentially like the classical result of Fefferman-Stein quoted above using the fact that we have appropriate control of the sharp maximal operator for the $M B M O$ case (cf. [6]).

Finally let us consider the proof of Theorem B. First we prove $\left(W_{1}^{k}, W_{2}^{k}\right)_{\theta}=W_{p_{\theta}}^{k}$, $1 / p_{\theta}=1-\theta / 2$.

Consider the bounded operator $L f=\Sigma_{|\alpha| \leqslant k} D^{\alpha} f, L: W_{p}^{k} \rightarrow L^{p}, 1 \leqslant p \leqslant \infty$. Interpolating the end point results $p=1,2$, we get $L:\left(W_{1}^{k}, W_{2}^{k}\right)_{\theta} \rightarrow L^{p_{\theta}}, 1 / p_{\theta}=1-\theta / 2$. Therefore, $\left(W_{1}^{k}, W_{2}^{k}\right)_{\theta} \subset W_{p_{\theta}}^{k}$.

On the other hand using type arguments as in the proof of Theorem $\mathrm{A}$ we have $\left(W_{1}^{k}, W_{2}^{k}\right)_{\theta, p_{\theta}} \subset\left(W_{1}^{k}, W_{2}^{k}\right)_{\theta}$. Thus the desired result now follows using (cf. [2,1]), $\left(W_{1}^{k}, W_{2}^{k}\right)_{\theta, p_{\theta}}=W_{p_{\theta}}^{k}$.

The well-known formula $\left(W_{p_{0}}^{k}, W_{p_{1}}^{k}\right)_{\theta}=W_{p_{\theta}}^{k}, 1 / p_{\theta}=(1-\theta) / p_{0}+\theta / p_{1}$, which is valid if $1<p_{0}, p_{1}<\infty$, allows us to use Wolff's theorem to fuse these end point results and conclude the proof of Theorem $B$.

ACKNOWLEDGEMENTS. This method was presented at the Conference of Interpolation spaces held at the University of Lund, August 1982 (cf. [7]). I wish to thank the participants for their helpful remarks. S. Janson in particular has a proof of the above results using his mixed reiteration theorems (to appear).

\section{REFERENCES}

1. C. P. Calderón and M. Milman, Interpolation of Sobolev spaces. The real method, Indiana Univ. J. (to appear).

2. R. DeVore and K. Scherer, Interpolation of linear operators on Sobolev spaces, Ann. of Math. 109 (1979), 583-599.

3. C. Fefferman and E. Stein, $H^{p}$ spaces of several variables, Acta Math. 129 (1972), 137-193.

4. S. Janson and P. Jones, Interpolation between $H^{p}$ spaces: The complex method, J. Functional Anal. 48 (1982), 58-80.

5. M. Milman, On interpolation of martingale $L^{p}$ spaces, Indiana Univ. Math. J. 30 (1981), 313-318.

6. _ Interpolation of martingale spaces and applications, 11 Sem. Bras. Anal. (Sao Carlos, 1980), pp. $92-108$. 
7. _ Interpolation of some concrete scales of spaces, Technical Report, Univ. of Lund, 1982.

8. J. Peetre, Sur la transformation de Fourier des fonctions à valeurs vectorielles, Rend. Sem. Mat. Padova 42 (1969), 15-26.

9. N. Rivière and Y. Sagher, Interpolation between $L^{\infty}$ and $H^{1}$, the real method, J. Functional Anal. 14 (1973), 401-409.

10. T. Wolff, A note on interpolation spaces, Harmonic Analysis, Lecture Notes in Math., vol. 908 , Springer-Verlag, Berlin, 1982.

Department of Mathematics, Southern Illinois University, Carbondale, Illinois 62901 